University of Nebraska - Lincoln

DigitalCommons@University of Nebraska - Lincoln

$3-1-2000$

\title{
Freezing transition of a strongly dipolar simple fluid
}

G.T. Gao

University of Nebraska-Lincoln

Xiao Cheng Zeng

University of Nebraska-Lincoln, xzeng1@unl.edu

Follow this and additional works at: https://digitalcommons.unl.edu/chemzeng

Part of the Chemistry Commons

Gao, G.T. and Zeng, Xiao Cheng, "Freezing transition of a strongly dipolar simple fluid" (2000). Xiao Cheng Zeng Publications. 52.

https://digitalcommons.unl.edu/chemzeng/52

This Article is brought to you for free and open access by the Published Research - Department of Chemistry at DigitalCommons@University of Nebraska - Lincoln. It has been accepted for inclusion in Xiao Cheng Zeng Publications by an authorized administrator of DigitalCommons@University of Nebraska - Lincoln. 


\title{
Freezing transition of a strongly dipolar simple fluid
}

\author{
G. T. Gao and X. C. Zeng \\ Department of Chemistry and Center for Materials Research and Analysis, University of Nebraska-Lincoln, Lincoln, Nebraska 68588
}

(Received 20 September 1999)

\begin{abstract}
The freezing transition of a strongly dipolar Lennard-Jones fluid is investigated using Monte Carlo simulation. It is found that the system undergoes a ferroelectric liquid-to-solid transition when cooled under a constant pressure. Near the triple point the stable solid phase is a body-centered orthorhombic ferroelectric crystal; a metastable ferroelectric solid phase with distorted hexagonal lattice structure is also discovered. To locate the freezing point, Gibbs free energies of the solid and liquid phase are determined using a thermodynamic integration method.
\end{abstract}

PACS number(s): 61.20.Ja, 61.20.Ne, 64.70.-p, 77.80.-e

The crystallization of liquid is a universal phenomenon, yet a central characteristic of crystallization is that it is highly nonuniversal [1]; that is, the location of the freezing point and the stable crystalline lattice selected upon freezing depend sensitively on the underlying molecular interactions. Computer simulations [2,3] have shed much light on the crystallization of a prototype nonpolar liquid-Lennard-Jones $[4,5]$. The latter is a widely used model of noble gases. It has been found that the stable solid phase of the Lennard-Jones system is a face-centered cubic (fcc) close-packed crystal, consistent with that of noble gases (except helium). However, much less is known about freezing of its simplest polar counterpart, Lennard-Jones particles carrying an embedded point dipole (or Stockmayer fluid [6]).

Examples of strongly dipolar systems include molecular systems such as hydrogen cyanide and complex fluid systems such as ferrofluids (colloidal suspensions of magnetic spherical particles) [7]. Upon freezing, solid hydrogen cyanide may select the orthorhombic structure, whereas the lattice structure of ferrofluids is less clear [8]. In any event, common to all strongly dipolar systems is that the symmetry of crystal structure manifests the subtle interplay between isotropic repulsion plus dispersive van der Waals attraction, which promotes cubic lattice structures, and anisotropic dipole-dipole interaction, which promotes orientational order.

The freezing transition of dipolar Lennard-Jones fluid has been studied by density-functional theory $[9,10]$, the best analytic theory of freezing to date [11]. Only fcc and bodycentered tetragonal (bct) structures were examined. Two issues remain under debate: (i) which lattice structure is selected upon freezing other than the fcc lattice structure (which is selected in high pressure or weak dipole limit)? (ii) Can a stable ferroelectric liquid phase intervene between the isotropic liquid phase and the crystal prior to the freezing transition $[9,10]$ ? In this Rapid Communication we report the results of computer simulations of a strongly dipolar Lennard-Jones system, which demonstrate that near the triple point the stable solid phase is a body-centered orthorhombic (bco) ferroelectric crystal and that the melt is a stable ferroelectric liquid.

The dipolar Lennard-Jones model is described by the pairwise interparticle potential

$$
u\left(r_{i j}\right)=4 \epsilon\left[\left(\frac{\sigma}{r_{i j}}\right)^{12}-\left(\frac{\sigma}{r_{i j}}\right)^{6}\right]+\frac{\vec{\mu}_{i} \cdot \vec{\mu}_{j}}{r_{i j}^{3}}-\frac{3\left(\vec{\mu}_{i} \cdot \vec{r}_{i j}\right)\left(\vec{\mu}_{j} \cdot \vec{r}_{i j}\right)}{r_{i j}^{5}}
$$

where $\sigma$ and $\epsilon$ are the Lennard-Jones diameter and well depth parameters, $\vec{\mu}_{i}$ is the dipole moment of the $i$ th particle, and $\vec{r}_{i j}$ denotes the vector connecting the $i$ th and $j$ th particles. We are particularly interested in the system with particles carrying a reduced dipole moment $\mu^{*}=\mu / \sqrt{\epsilon \sigma^{3}}$ $=2.5$. For convenience, we will use the reduced temperature $T^{*}=k_{B} T / \epsilon$, density $\rho^{*}=\rho \sigma^{3}$, and pressure $p^{*}=p \sigma^{3} / \epsilon$ to specify thermodynamic states of the system.

In the dipolar system, the onset of orientational order can be monitored through two orientational order parameters $[12,13]$. (1) The nematic order parameter $S$, defined as an ensemble average of the largest eigenvalue of the secondrank tensor $\stackrel{\leftrightarrow}{Q}=(1 / N) \sum_{i=1}^{N} \frac{1}{2}\left(3 \hat{u}_{i} \hat{u}_{i}-\stackrel{\leftrightarrow}{I}\right)$, where $\hat{u}_{i}$ is the unit vector along the direction of the dipole $\vec{\mu}_{i}$, and (2) the polarization order parameter $P=\left\langle 1 / N\left|\sum_{i=1}^{N} \hat{u}_{i} \cdot \hat{d}\right|\right\rangle$, where $\hat{d}$ is the so-called instantaneous director, which is the unit eigenvector associated with the largest eigenvalue of $\stackrel{\leftrightarrow}{Q}$. Note that $S>0$ indicates the appearance of the nematic order, whereas $P>0$ signifies the onset of ferroelectric order. We also monitored the positional order of the system through the pair correlation functions $g(r), g_{\|}\left(r_{\|}\right)$, and $g_{\perp}\left(r_{\perp}\right)$ [13-15]. The latter two are the longitudinal and transverse pair correlation functions which measure positional correlations in directions parallel and perpendicular to the director $\hat{d}$.

Two series of isothermal-isobaric Monte Carlo (MC) simulations were performed, after the manner of Weis and Levesque [13]. In the first series a cubic box is chosen as the simulation cell, where it contains 500 particles; the initial configuration is an fcc crystal at a high reduced temperature $(2.0-2.5)$. In the second series, the initial configuration is a crystal but at a low reduced temperature $(0.5-0.8)$. A rectangular parallelepiped is chosen as the simulation cell where it contains either 432 or 500 particles, depending on whether the body-centered [including bct, bco, and body-centered cubic bcc], or the fcc and hexagonal close-packed (hcp) lattice is selected as the initial structure. All three lengths of the parallelepiped are allowed to change independently so that the solid is allowed to adapt a favorable lattice structure at 


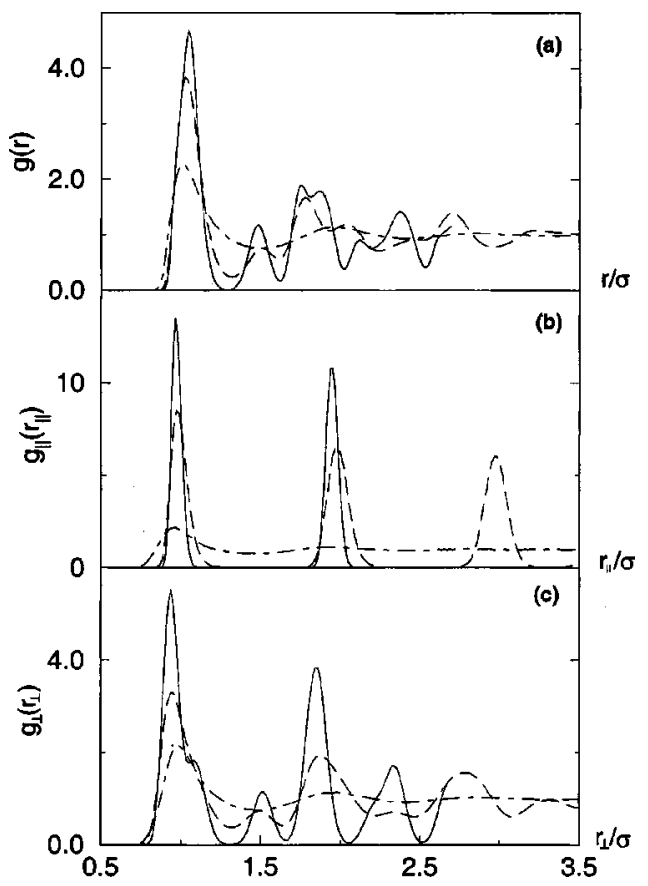

FIG. 1. (a) Radial distribution function $g(r)$; (b) longitudinal correlation function $g_{\|}\left(r_{\|}\right)$; (c) transverse correlation function $g_{\perp}\left(r_{\perp}\right)$. The dotted-dashed lines are for the isotropic liquid at $T^{*}$ $=2.5$ and $p^{*}=5.0$; the dashed lines are for the bco solid at $T^{*}$ $=1.2$ and $p^{*}=5.0$, resulting from the first series of isothermalisobaric MC simulations; the solid lines are for the bco solid at $T^{*}=0.7$ and $p^{*}=5.0$, resulting from the second series of simulations.

the low temperature. The long-range dipolar interactions are calculated using the Ewald sum method with the "tin-foil" boundary $[16,17]$. Periodical boundary conditions are imposed in all simulations.

We consider three isobaric conditions: $p^{*}=0.0,3.0$, and 5.0. At each pressure and a given temperature, $30000 \mathrm{MC}$ cycles were used for equilibration, followed by another 30000 or more MC cycles for calculation. Starting the first simulation at $p^{*}=5.0$ and $T^{*}=2.5$ with the orientation of dipoles set randomly on the lattice sites, the fcc lattice transforms into a liquid after several thousand MC cycles. The correlation functions of the liquid at $p^{*}=5.0$ and $T^{*}=2.5$ are shown in Figs. 1(a)-1(c) (dotted-dashed lines). Subsequent runs at a lower temperature always start from the final configuration of the preceding run at a higher temperature. Figures 2(a)-2(c) display the temperature dependence of the reduced potential energy per particle $U^{*} / N$ and the two order parameters $S$ and $P$, respectively. At high temperatures both $S$ and $P$ are close to 0 , indicating that the liquid phase is isotropic with no orientational order. As the temperature is lowered, $U^{*} / N$ decreases gradually while $S$ and $P$ increase first slowly and then rapidly, indicating the appearance of both nematic and ferroelectric orders in the system $[18,19]$. At $T^{*} \sim 1.0, U^{*} / N$ exhibits a sudden drop while both $S$ and $P$ show a sudden jump, manifesting a strong first-order transition. Graphical analysis of a typical configuration of the simulation reveals that the post-transition phase exhibits a bco lattice structure. An example of the pair correlation functions for the post-transition state at $T^{*}=1.2$ and $p^{*}=5.0$ is displayed in Figs. 1(a)-1(c) (dashed lines).

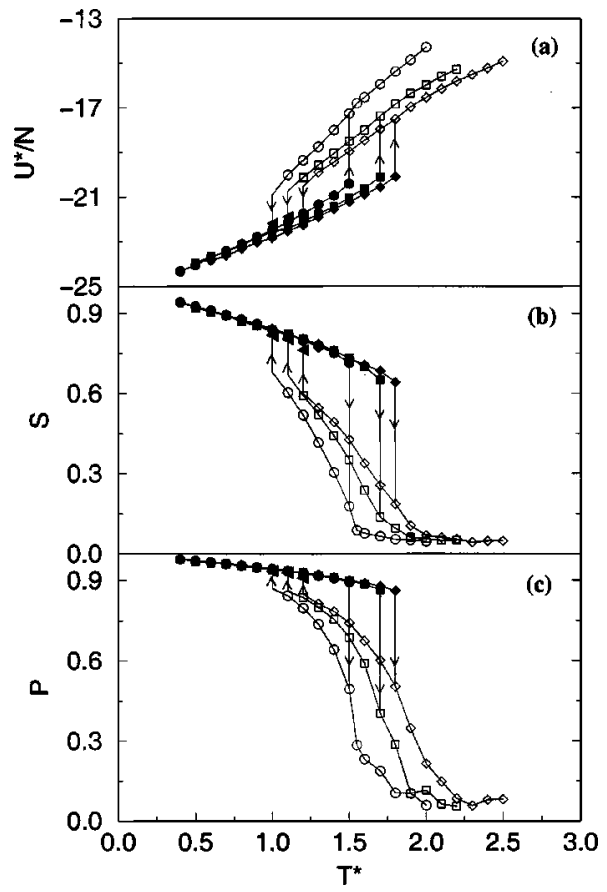

FIG. 2. Temperature dependence of (a) the reduced potential energy per particle $\left(U^{*} / N \equiv U / N \epsilon\right)$, (b) nematic order parameter $S$, and (c) polarization order parameter $P$ for liquid phase (open symbols) and bco solid phase (closed symbols) at three isobaric conditions: $p^{*}=0.0$ (circle), 3.0 (square), and 5.0 (diamond).

To further validate this observation, the second series of simulations was performed starting respectively with three body-centered lattice structures: bcc, bct, and bco, and with two close-packed lattice structures, fcc or hcp. Both random and ordered initial orientations of dipoles are used. For the ordered one, the orientation of all dipoles either is along the [001] (for bcc, bct, and bco) or the [110] direction (for fcc and hcp) because these directions are parallel to the closepacked planes of the crystal. After equilibration at a low temperature $T^{*}=0.5-0.8$, the systems with bcc or bet structures all quickly transform into the bco structure with a high degree of orientational order along [001] direction [Fig. 3(a)] and the system with fcc structure also slowly evolves into the bco lattice structure. This indicates that at $T^{*}=0.5-0.8$ the $\mathrm{bcc}$, bct, and fcc solid phases are unstable relative to the bco (a)

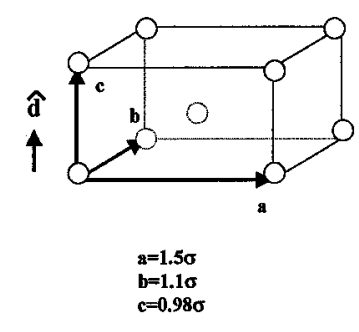

(b)

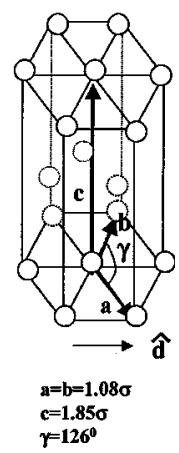

FIG. 3. (a) Unit cell of the bco solid at $T^{*}=0.7$ and $p^{*}=5.0$; (b) A unit cell of the distorted hexagonal solid at $T^{*}=0.8$ and $p^{*}=0.0$. 
solid, regardless of the initial orientation of dipoles. However, the system starting with the hcp structure and with random dipole orientation does not transform to a bco structure but to a glassylike phase with pair correlation functions similar to those of liquid at high temperatures (Fig. 1). In this case the system is trapped in a metastable liquid state. More interestingly, the system starting with hcp structure and ordered dipole orientation ends up with a distorted hexagonal lattice structure, where the distortion is a contraction along the direction of the director (the [110] direction) so that the angle $\gamma$ between $a$ and $b$ axes is greater than $120^{\circ}$ [see Fig. 3 (b)]. We will later show from free energy calculations that near the triple point the distorted hexagonal solid is a metastable phase, whereas the bco solid is the stable one.

Figures 1(a)-1(c) also display the correlation functions for the bco solid state at $T^{*}=0.7$ and $p^{*}=5.0$ (solid lines). Note that the solid and dashed lines in Fig. 1 show similar features. From the longitudinal and transverse correlation functions we determine the lattice constants $(a, b, c)$ of the unit cell. Specifically, for the solid at $T^{*}=0.7$ and $p^{*}=5.0$, the peak separation in the $g_{\|}\left(r_{\|}\right)$curve is 0.98 . This gives the lattice constant $c=0.98$ [see Fig. 3(a)]. In the $g_{\perp}\left(r_{\perp}\right)$ curve the position of the first peak is $r_{\perp} \sim 0.93$, consistent with the length $\sqrt{a^{2}+b^{2}} / 2$; the second and third peaks occur at $r_{\perp}$ $\sim 1.1$ and 1.5 , consistent with the lattice constants $a=1.5$ and $b=1.1$; the position of the fourth peak is at $r_{\perp} \sim 1.86$, consistent with the distance between atoms along [110] direction, $\sqrt{a^{2}+b^{2}}$.

When the temperature is raised from 0.5 to 0.8 we find $U^{*} / N$ exhibits an abrupt jump for $T^{*}>1.5$, an indicator of a melting transition, and $S$ and $P$ also show an abrupt drop (see Fig. 2). Indeed, the hysteresis behavior manifests a strong first-order phase transition.

Why is the bco lattice structure selected over the fcc structure upon freezing? This is due to the strong attractive interactions between dipolar particles along the [001] direction. As a result, the lattice constant $c$ becomes the smallest among $a, b$, and $c$. In fact, $c$ is nearly a constant, insensitive to the decrease of temperature, whereas $a$ and $b$ can vary considerably. Then, why is the bco lattice structure selected over the bct structure? Namely, why is $a$ not equal to $b$ ? Careful analysis of the data indicates that although $a$ and $b$ varies considerably as the temperature decreases, the distance $\sqrt{a^{2}+b^{2}+c^{2}}$ remains more or less a constant, reflecting strong attractive interactions between the body-centered particle with its nearest neighbors along [111] directions. In addition, the solid prefers to hold a smaller volume (which is proportional to $a b c$ ) as the temperature decreases. As a result, a large difference between $a$ and $b$ is preferred provided that $c$ remains a constant.

In order to determine the exact location of the transition points we evaluate the Gibbs free energy $G=A+p V$ of both liquid and solid bco and distorted hexagonal) phases, where $A$ is the Helmholtz free energy and $V$ is the volume of the system. To this end, we adopted a thermodynamic integration method [20]. For the liquid, the ideal gas was chosen as the reference system. Simulations were carried out first on an isotherm $\left(T_{1}^{*}=3.0\right)$ from $\rho^{*} \approx 0$ (ideal gas) to a desired density $\rho^{*}$ and then on an isochore $\left(\rho^{*}\right)$ from $T_{1}^{*}$ to a desired temperature $T^{*}$.

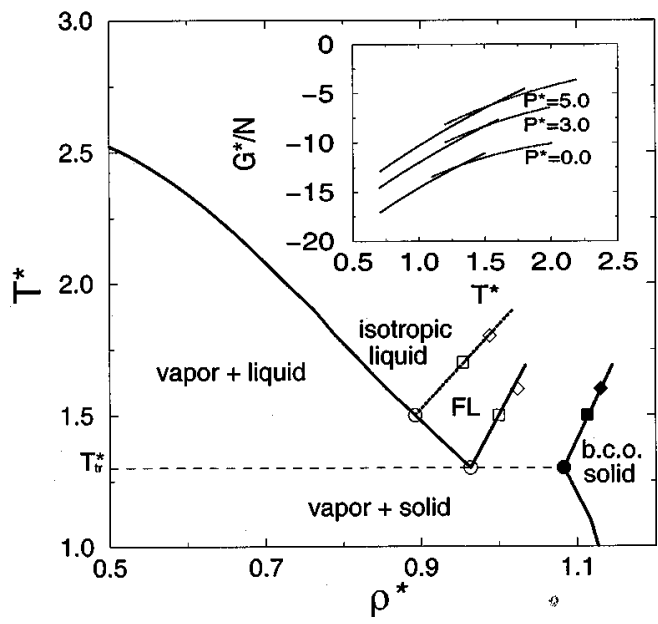

FIG. 4. Density-temperature phase diagram of the strongly dipolar Lennard-Jones system with $\mu^{*}=2.5$. FL stands for the ferroelectric liquid and $T_{t r}^{*}$ denotes the reduced temperature of triple point. Inset: Temperature dependence of the reduced Gibbs free energy per particle for the bco solid (solid lines) and ferroelectric liquid (dotted lines) at three isobaric conditions. The crossing point of the liquid and solid free energy curves gives the freezing temperature at the corresponding pressure.

For the solid, the reference system is chosen to be an Einstein crystal [21] with a constraint on the direction of dipoles. The particle positions and dipole orientations $\left(\vec{r}_{0}^{N}, \vec{\mu}_{0}^{N}\right)$ of the reference system are obtained by minimizing the potential energy [22]; the (local) potential energy minimum is denoted by $U_{0}\left(\vec{r}_{0}^{N}, \vec{\mu}_{0}^{N}\right)$. Note again that for the bco solid the orientation of all dipoles is parallel to the [001] direction ( $c$ axis), whereas for the distorted hexagonal solid the orientation is parallel to the [110] direction. The potential energy of the reference system can then be written as $U_{R}$ $=U_{0}\left(\vec{r}_{0}^{N}, \vec{\mu}_{0}^{N}\right)+\sum_{i=1}^{N} \alpha\left(\vec{r}_{i}-\vec{r}_{i 0}\right)^{2}+\sum_{i=1}^{N} \gamma\left(1-\hat{\mu}_{i} \cdot \hat{d}\right)$, where the second term is the harmonic potential with a spring constant $\alpha$ and the third term constrains the system to maintain ferroelectric order [23]. The free energy difference between the solid and the reference system can be obtained from the simulations [20].

Gibbs free energies per particle for both the bco and the distorted hexagonal solids were computed for the three isobaric conditions. It turns out that the Gibbs free energy per particle for the bco solid is typically a few tenths of $k_{B} T$ lower than that for the distorted hexagonal solid; that is, the distorted hexagonal solid is metastable at least for $0.0 \leqslant p^{*}$ $\leqslant 5.0$. In the inset of Fig. 4 we plot the reduced Gibbs free energy per particle $G^{*} / N=G / N \epsilon$ as a function of $T^{*}$ for both liquid and bco solid, where the crossing point of the free energy curves gives rise to the freezing temperature $T_{f}^{*}$, which is about $1.31,1.50$, and 1.56 for three isobaric conditions $p^{*}=0.0,3.0$, and 5.0, respectively. Note that the freezing temperature $T_{f}^{*}=1.31$ at $p^{*}=0.0$ can be considered approximately as the triple point temperature $T_{t r}^{*}$ because near the triple point the vapor pressure is almost zero. We also reexamined the ferroelectric fcc, bct, and bcc solid phases near the triple point and found all of them are unstable relative to the bco phase.

In Fig. 4, we show the density-temperature phase dia- 
gram. We note that using a density functional theory Klapp and Forstmann [10] predicted that the ferroelectric liquid phase is metastable and it is surpassed by a ferroelectric bct phase. Our simulations show that the ferroelectric liquid can be a stable phase and upon freezing it transforms into a bco ferroelectric solid phase rather than a bct ferroelectric solid phase. As we are mainly interested in the existence of ferroelectric liquid phase, no attempt has been made to determine the order of isotropic-to-ferroelectric liquid phase transition. It seems the transition could be either second order or weakly first order (see Fig. 2). A dotted line, which denotes the liquid states with a polarization $P=0.5$, is used in Fig. 4 to show schematically the existence of a transition from the isotropic to ferroelectric liquid phase [24].

In summary, we studied the freezing transition of a strongly dipolar Lennard-Jones system using Monte Carlo simulation methods. We find that the system undergoes a ferroelectric liquid-to-bco ferroelectric solid first-order phase transition when cooled at constant pressure in the range 0.0 $\leqslant p^{*} \leqslant 5.0$. A metastable ferroelectric distorted hexagonal solid is also discovered. In passing, we note that for the nonpolar Lennard-Jones system recent studies using computer simulation and density functional theory $[25,26]$ have shown that the isotropic liquid-to-fcc solid transition likely go through two steps: a homogeneous nucleation of bcc nuclei followed by a structural transformation to fcc crystallites. The reason for this behavior is that the barrier to the homogeneous nucleation of the fcc phase is higher than that of the bcc metastable phase. As a result, nuclei of crystal may exhibit both bcc and fcc characteristics. In contrast, for the strongly dipolar Lennard-Jones the stable lattice structure is neither fcc nor bcc but bco (at least for $0.0 \leqslant p^{*} \leqslant 5.0$ ). It is possible that the barrier to the homogeneous nucleation of a bco crystal is very low (if not the lowest) compared to other metastable or unstable phases so that homogeneous nucleation of the bco crystal can be observed directly from the computer simulation.

We thank Professor D. J. Diestler and Mr. K. J. Oh for valuable discussions. X.C.Z. thanks the NSF and ONR for support of this work.
[1] D. W. Oxtoby, Nature (London) 347, 725 (1990).

[2] D. Frenkel and J. P. McTague, Annu. Rev. Phys. Chem. 31, 491 (1980).

[3] B. B. Laird and A. D. J. Haymet, Chem. Rev. 92, 1819 (1992).

[4] J. P. Hansen and L. Verlet, Phys. Rev. 184, 151 (1969).

[5] A. Rahman, M. J. Mandel, and J. P. McTague, J. Chem. Phys. 64, 1564 (1976).

[6] W. H. Stockmayer, J. Chem. Phys. 9, 398 (1941).

[7] R. Rosensweig, Ferrohydrodynamics (Cambridge University Press, Cambridge, England, 1985).

[8] W. Luo, S. R. Nagel, T. F. Rosenbaum, and R. E. Rosensweig, Phys. Rev. Lett. 67, 2721 (1991).

[9] B. Groh and S. Dietrich, Phys. Rev. E 54, 1687 (1996).

[10] S. Klapp and F. Forstmann, Europhys. Lett. 38, 663 (1997).

[11] See, for example, R. Evans, in Fundamentals of Inhomogeneous Fluids, edited by D. Henderson (Dekker, New York, 1992), and references therein.

[12] D. Wei and G. N. Patey, Phys. Rev. A 46, 7783 (1992).

[13] J. J. Weis and S. Levesque, Phys. Rev. E 48, 3728 (1993).

[14] A. Stroobants, H. N. W. Lekkerkerker, and D. Frenkel, Phys. Rev. A 36, 2929 (1987).

[15] E. de Miguel, L. F. Rull, M. K. Chalam, and K. E. Gubbins, Mol. Phys. 74, 405 (1991).
[16] S. W. de Leeuw, J. W. Perram, and E. R. Smith, Proc. R. Soc. London, Ser. A 373, 27 (1980).

[17] Specifically, the $\alpha$ parameter in the Ewald sum was chosen such that $\alpha L_{\text {min }}=5.75$, where $L_{\text {min }}$ is the minimum length among three lengths of the simulation box.

[18] D. Wei and G. N. Patey, Phys. Rev. Lett. 68, 2043 (1992).

[19] J. J. Weis, S. Levesque, and G. J. Zarragoicoechea, Phys. Rev. Lett. 69, 913 (1992).

[20] D. Frenkel and B. Smit, Understanding Molecular Simulation (Academic Press, San Diego, 1996).

[21] D. Frenkel and A. J. C. Ladd, J. Chem. Phys. 56, 3188 (1984).

[22] F. H. Stillinger and T. A. Weber, Phys. Rev. A 25, 978 (1982).

[23] We found that if $\alpha$ is chosen large enough to keep the solid intact the final result of free energy is insensitive to the value of $\alpha$. The parameter $\gamma$ should also be chosen large enough such that the two order parameters $S$ and $P$ of the Einstein crystal is close to those of the bco or hexagonal solid.

[24] M. J. Stevens and G. S. Grest, Phys. Rev. E 51, 5976 (1995).

[25] P. R. ten Wolde, M. J. Ruiz-Montero, and D. Frenkel, Phys. Rev. Lett. 75, 2714 (1995).

[26] Y. C. Shen and D. W. Oxtoby, Phys. Rev. Lett. 77, 3585 (1996). 